\title{
Medical metrology studies at Tübitak UME
}

\author{
Karaböce Ba , Gülmez Y, Akgöz M, Kaykısızlı H, Yalçınkaya B and Dorosinskiy L \\ Ulusal Metroloji Enstitüsü-National Metrology Institute of Turkey \\ Barış Mah. Dr.Zeki Acar Cad. No: 1 Gebze 41470 Kocaeli Turkey
}

\begin{abstract}
Calibration and testing of medical devices are one of the most important and critical issue in metrology field. Although metrological links has been well established for measurements in technical and military field, metrology link is not powerful enough for measurements in medical field. For the medical device industry and applications in health sector, nothing counts more than the safety of a patient. Therefore all hospitals \& medical equipment manufacturers have to perform periodic testing and calibration of equipments, as a quality control regimen that guarantees the reliability of medical devices. Test, measurement and calibration of bio-medical equipments is becoming increasingly significant, when accuracy in diagnosis and effectiveness in treatment is required. A feasibility study has been carried out and a report was published last year at TÜBİTAK UME (The Scientific and Technological Research Council of Turkey, National Metrology Institute) in order to outline the current situation in the country. A five year roadmap and a a plan were programmed for providing of reliability and metrological traceability in medical measurements. Medical metrology research laboratory has been established and a number of medical device design projects were initiated.
\end{abstract}

\section{Introduction}

According to the information from personal communication and limited publications, it was observed that the meaning of calibration and metrology are not known by medical device users. Then, important issue becomes to educate and train medical people who are engaged in use of medical devices and measurements. Trainings must be given to medical device users in order to have them aware of metrological terms, importance and role of metrology and methodology of measurement and calibration.

Medical devices are calibrated against national or international standards by accredited laboratories. Reference devices/standards are calibrated against reference standards which are traceable to SI and derived SI units. But unfortunately, some improper applications were observed for medical device calibration procedures. Only checking some switches, buttons, lamps/LEDs, numbers and displays is considered test or calibration. Even some calibration certificates and labels were prepared without performing any measurement or calibration of medical devices.

Test, measurement and calibration are critically important in achieving quality control of the highest standard in medical equipment. Written national and international standards aim to control this field including IEC60601 series, EN61010 series, ISO 15189, ISO 13485, ANSI/AAMI SP10 and more [1-5].

Most of the entire range of medical equipments including defibrillators Pulse Oxymeters, Infusion pumps, Patient Simulators, ventilators, Fetal Monitors, Patient monitors are extremely critical devices. All this

\footnotetext{
${ }^{a}$ Corresponding author: baki.karaboce@tubitak.gov.tr
}

devices must be tested, measured or calibrated by trained engineers or device users with certified reference materials. A measurement or calibration report must be concluded by documenting the test results and issuing a calibration report [6].

An example of blood sugar measurement errors presented by NIST researchers show the importance of the accuracy in measurements [7]. If blood sugar is measured higher than its true value, medical doctor prescribes insulin. Or if blood sugar is measured lower than its true value, treatment will not be planned. In both cases, patient either will face the risk of injury/death or will consume unnecessary drug and lose money. When the accuracy is increased or uncertainty is decreased in blood sugar measurement, money will be saved and the quality of life will increase as can be seen in Figure. 1.

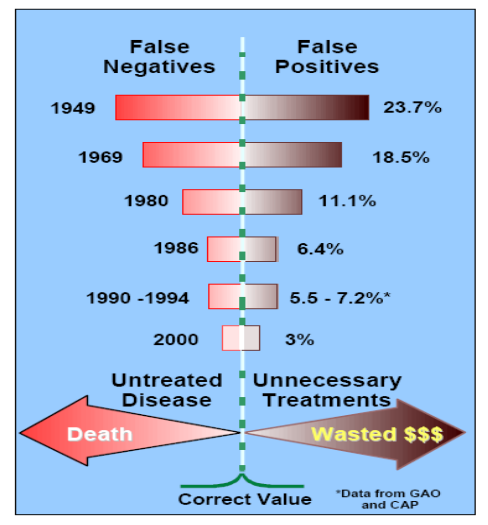

Figure 1. Importance of accuracy in blood sugar measurement Therefore, a survey should be performed on this subject in order to evaluate the current situation and to 
elucidate the use standards and legislations in the country, a master plan and a roadmap should be prepared and conducted.

\section{Methodology}

A survey/research group has been constituted from different disciplines such as physicist, chemist, biologist and electric/electronics engineers. Two year work plan was prepared and implemented with the support of TÜBİTAK UME management.

All written standards/legislations and publications were compiled and studied. Medical metrology studies and current laboratories of other metrology institutes were searched in order to see the situation in the Europe and in the world. Many interviews with researchers engaged in medical devices and measurements from ministry of health, universities and private laboratories have been conducted.

Personal interviews and meetings with official institutions, organizations, universities, laboratories and calibration firms were arranged as well as literature review in order to determine current situation in the medical field of metrology in Turkey. One of the major stakeholder and supporter is Ministry of Health, Turkish Medicine and Medical Device Division (TITCK) in feasibility studies. Very close cooperation and collaboration has been established with TITCK for "Regulation of medical device test, measurement and calibration". Discussions on the subject were made with Turkish Accreditation Agency, (TÜRKAK) for accreditation and Turkish Atomical Energy Authority (TAEK) for ionizing radiation measurements.

Studies in universities (Boğaziçi University, Gazi University, Ankara Military Medicine Academy (GATA), İstanbul University), in medical metrology field were investigated briefly, in order to create a research environment for medical device design and production.

Services in the Medical calibration laboratories and hospitals have been searched and discussed. Presentations were made in medical related congresses in order to explain the importance of metrology in medical measurements. Fairs have been participated in order to see the current situation in medical devices traceability. After evaluating the results of all these surveys, it has been decided that a medical metrology laboratory should be establishment at UME.

\subsection{Medical Metrology Road Map}

A five year project has been initiated; a work plan and a roadmap were prepared and implemented as it is seen in Figure 2.

Starting point is ensuring the reliability of measurements, analyzers, simulators and phantoms used in the health sector. The concept of measurement traceability provides probably the most important strategy to achieve standardization in medical metrology and is aimed at accurate and comparable measurement results regardless of the method, the measurement procedure (test kit) and the laboratory where analyses are carried out.

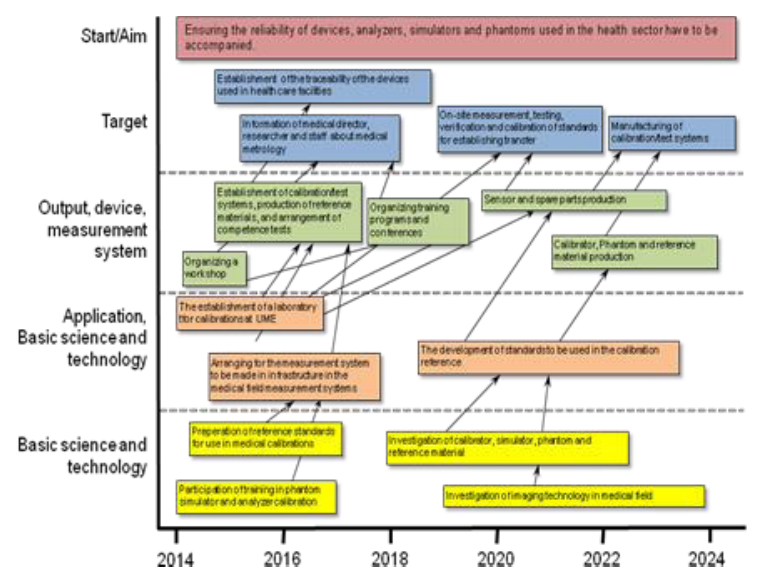

Figure 2. Medical metrology road map at UME

Targets were maintained like; establishment of the traceability of the devices used in health care facilities, information of medical director, researcher and staff about medical metrology, on-site measurement, testing, verification and calibration of standards for establishing transfer and manufacturing of calibration/test systems within ten years.

Then, a number of deliverables were aimed such as organizing a workshop, establishment of calibration/test systems, production of reference materials, and arranging proficiency tests, organizing training programs and conferences, sensor, spare parts , calibrator, phantom and reference material production.

In-Vitro Diagnostics (IVD) Directive of the European Union stipulates that values assigned to calibrators and control materials must be traceable to reference materials and/or reference methods of a higher metrological order $[8,9]$. Subsequently, the Joint Committee for Traceability in Laboratory Medicine (JCTLM) was established in 2002. The International Federation of Clinical Chemistry and Laboratory Medicine (IFCC), the Bureau International des Poids et Mesures (BIPM) and the International Laboratory Accreditation Cooperation (ILAC)have contributed to the JCTLM. Two working groups (WG) have been established within the JCTLM. WG1 deals with reference materials and reference measurement procedures that are listed on the BIPM website [10]. The task of $\mathrm{WG} 2$ is to list reference measurement laboratories. The first list of reference measurement services provided by reference calibration laboratories was published in 2007.

Metrology institutes from all over the world have some service or organization for traceability of medical measurements and devices. For example, USA, German and French metrology institutes have medical device sections or laboratories in order to establish the traceability of medical measurements and devices [1115]. NIST (National Institute of Standards and Technology) from USA has same subject areas like; bioscience and health, medical devices, diagnostics, standard reference materials and medical devices metrology and standards. Similarly, PTB (The 
Physikalisch-Technische Bundesanstalt) from Germany has medical metrology, standards for medical instrumentation and biomedical optics sections. LNE (Laboratoire national de métrologie et d'essais) from France has medical metrology facilities and produces certified reference materials.

\subsection{Traceability in Medical Measurements and Calibrations}

As demonstrated in Figure 3, traceability of a value attributed to a routine sample, a calibrator or a control material is established by a series of comparative measurements using measurement procedures and reference materials in a chain of increasing hierarchical order. An inevitable precondition for establishing traceable results to calibrators and control materials is the specificity of the measurement procedures applied. Results of measurement cannot be traceable if the procedure applied partially detects components which are not consistent with the definition of the measurand.

For example a traceability chain can be established as follows; audiometers, hearing aids, headphones and other audiology devices are calibrated by artificial ear and mastoid which are traceable to reference microphones and accelerometers. They all referenced by reciprocity calibration system and accelerometer calibration system by laser interferometry. Sound pressure level as $\mathrm{dB}$, vibration level as $\mathrm{m} / \mathrm{s}^{2}$ and frequency as $\mathrm{Hz}$ are measurands in the calibration according to IEC 603181:2009 and IEC 60373:1990 [16,17].

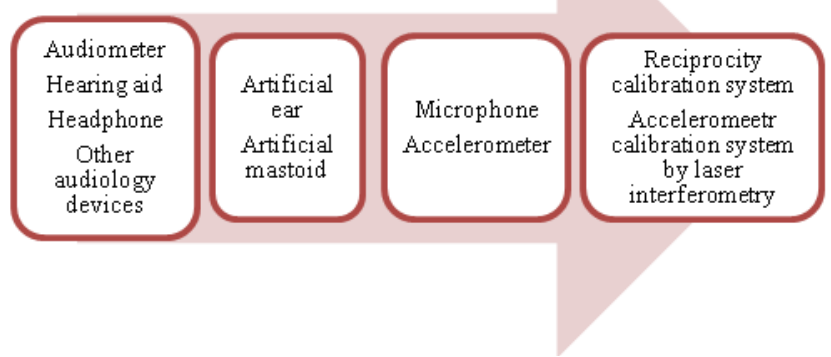

Figure 3. Traceability chain for audiometric devices

\section{Results and Discussion}

Without any opposition, all universities, institutions, laboratories and companies agree with the importance of medical metrology. They all requested and advised UME to establish calibration and measurement systems in medical field and to stimulate regulatory role.

Therefore TUBITAK UME has established a Medical Metrology Research Laboratory and has begun to implement 5 year development plan based on the road map.

\subsection{Short Term Plans}

Calibration systems for patient simulator, defibrillator analyzer, infusion pump, electro-surgery analyzer, electrical safety analyzer, pulse-oximetry analyzer and gas flow analyzer calibration systems have been set up in the Medical Metrology Research and Calibration Laboratory at TUBITAK UME as it is seen in Figure 4.

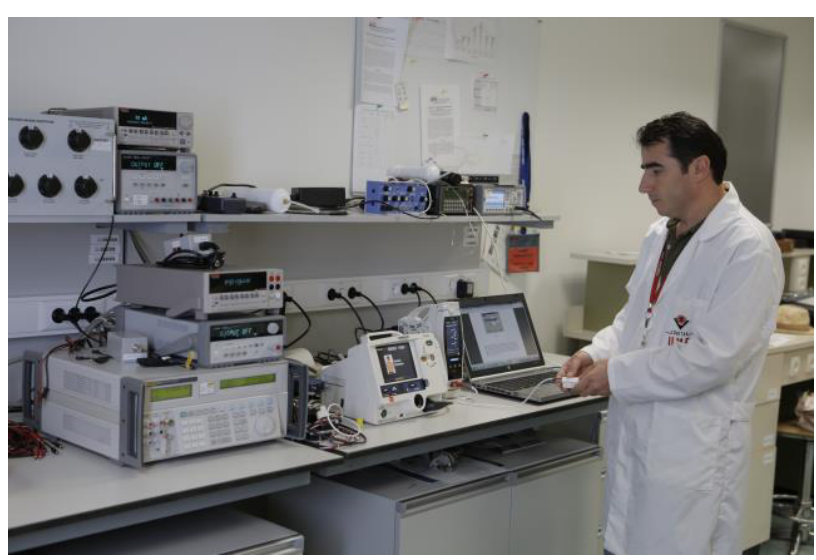

Figure 4. Measurement and calibration systems in Medical Metrology Research Laboratory (only some part of laboratory is seen here

\subsection{Mid Term Plans}

Research projects will be initiated in order to design and produce IR temperature reference device for developing ear thermometers, blood pressure meter calibration system and autoclave calibration system, in the next 3 years. Other similar projects will be initiated with the collaboration of interested stakeholders (universities, institutes etc).

Moreover, production of certified reference materials (CRM) was planned and several CRM production projects started, as seen in Figure 5. Proficiency tests were organized in the first year.

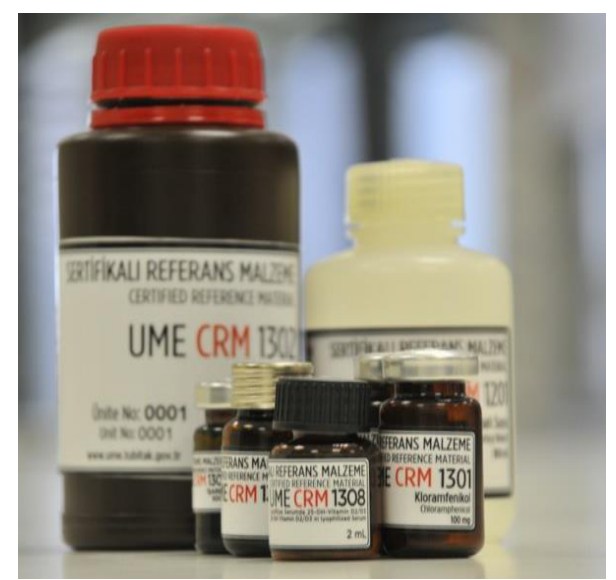

Figure 5. Reference Materials produced at UME Chemical Laboratoies within the medical metrology project 


\subsection{Long Term Plans}

Proficiency tests within the laboratories in the country have been planned for each year for different analytes. Design and development of prototypes of reference phantoms for MRI (magnetic resonance imaging) and ultrasound systems. Either supporting SME's for development of automatized blood autoanalyzer or designing itself is one of the future plans within five years. Another research study has been planned in imaging techniques. Multi-element HIFU array system design and production will be initiated.

Therefore most of the critical and mostly used medical calibrators will be included in the traceability chain throughout UME reference standards as can be seen in Figure 6.

Place the figure as close as possible after the point where it is first referenced in the text. If there is a large number of figures and tables it might be necessary to place some before their text citation. If a figure or table is too large to fit into one column, it can be centred across both columns at the top or the bottom of the page.

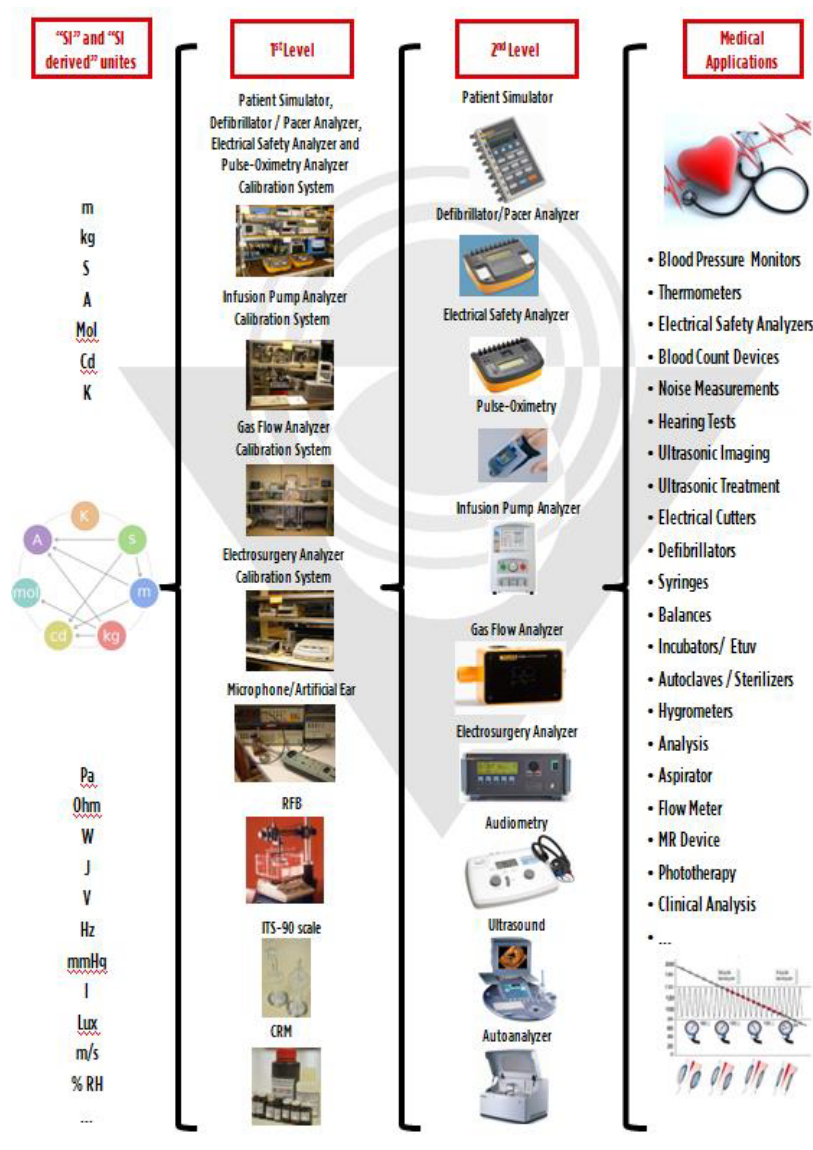

Figure 6. Measurement and calibration

\section{Conclusion}

A medical metrology research and calibration laboratory has been established at TÜBİTAK UME. Medical calibrators, analyzers and simulators used in medical device calibrations are now traceable to UME reference standards. Training programs were prepared for laboratory researchers in medical metrology field. A number of medical device/calibrator design and prototype manufacturing have been planned.

Most of the SME's don't have research and development section as this cost much. They generally need small hints and design tricks in development of medical devices that has usually multi-parameter measurement features. As metrology institutions are multidisciplinary structures, they can support medical device developers like SME's. Few projects have been initiated within the scope of medical metrology research activities at TÜBITAK UME. This vision may be an example for other national metrology institutions.

\section{Acknowlegdement}

Authors thank to Dr. Fatih Üstüner for his support to medical metrology activities at TUBITAK UME

\section{References}

[1] IEC 60601 Series of technical standards for the safety and effectiveness of medical electrical equipment

[2] BS EN 61010-1:2010 Safety requirements for electrical equipment for measurement, control, and laboratory use. General requirements

[3] International Organisation for Standardisation. ISO 15189:2007 Medical Laboratories - Particular requirements for quality and competence. Geneva, Switzerland, 2007.

[4] International Organisation for Standardisation. ISO 13485:2003. Medical devices - Quality management systems - Requirements for regulatory purposes. Geneva, Switzerland, 2008.

[5] ANSI/AAMI SP10:2002 Manual, electronic or automated sphygmomanometers. Association for the Advancement of Medical Instrumentation. Arlington VA. 2002.

[6] ISO/IEC 17025:2005. General requirements for the competence of testing and calibration laboratories.Geneva, Switzerland: ISO; 2005 .

[7] Symposium Celebrating $10^{\text {th }}$ Anniversary of the CIPM MRA October 8-9, 2009 and ECONOMIC IMPACT OF METROLOGY, Dr Franz Hengstberger - Member of the CIPM CIPM: International Committee of Weights and Measures

[8] L. Siekmann, Requirements for Reference (Calibration) Laboratories in Laboratory Medicine, Clin Biochem Rev. Nov 2007; 28(4): 149-154

[9] Directive 98/79/EC of the European Parliament and of the Council of 27 October 1998 on in vitro diagnostic medical devices. Official Journal of the European Communities 1998; L 331.

[10] BIPM: Bureau International des Poids et Mesures. [Accessed 14 September 2007]. www.bipm.org.

[11] http://www.nist.gov/medical-devices-portal.cfm

[12] http://www.nist.gov/el/isd/medical_devices.cfm

[13] http://www.ptb.de/cms/en/fachabteilungen/abt8.html

[14] http://www.lne.eu/en/markets/medical-health.asp

[15] M.Do Céu Ferreira, The role of metrology in medical devices, Instituto Português da Qualidade, Portugal, OIML Bulletin Volume L II, Number 4, October 2011

[16] IEC 60318-1:2009 Electroacoustics - Simulators of human head and ear- Part 1: Ear simulator for the measurement of supra-aural and circumaural earphones

[17] IEC 60373:1990 Mechanical coupler for measurements on bone vibrators 